\title{
Polarization dependent coupling of surface plasmon on a one-dimensional Ag grating with an InGaN/GaN dual-quantum-well structure
}

\author{
Kun-Ching Shen \\ Graduate Institute of Electronics Engineering, National Taiwan University, 1, Roosevelt Road, Section 4, \\ Taipei 10617, Taiwan, Republic of China \\ Cheng-Yen Chen, Chi-Feng Huang, Jhy-Yang Wang, Yen-Cheng Lu, \\ Yean-Woei Kiang, and C. C. Yang ${ }^{\text {) }}$ \\ Institute of Photonics and Optoelectronics and Department of Electrical Engineering, National Taiwan \\ University, 1, Roosevelt Road, Section 4, Taipei 10617, Taiwan, Republic of China \\ Ying-Jay Yang \\ Graduate Institute of Electronics Engineering and Department of Electrical Engineering, National Taiwan \\ University, 1, Roosevelt Road, Section 4, Taipei 10617, Taiwan, Republic of China
}

(Received 27 September 2007; accepted 7 December 2007; published online 3 January 2008)

\begin{abstract}
The authors report the observation of a polarization-dependent surface plasmon (SP) feature on a one-dimensional Ag-grating structure through the SP coupling with an InGaN/GaN dual-quantum-well structure closely below the metal grating. Polarized photon output is observed because only the momentum matching condition of the SP mode propagating in the direction perpendicular to the grating grooves can be reached through the diffraction of the fabricated grating and, thus, the SP radiation efficiency is significantly enhanced only in this polarization. The dispersion curve of the observed SP mode shows a group velocity of $2.4 \times 10^{8} \mathrm{~m} / \mathrm{s}$, which manifests the SP characteristics in the air/Ag/GaN grating structure. (c) 2008 American Institute of Physics. [DOI: 10.1063/1.2829794]
\end{abstract}

Due to the existence of a strong local field, the coupling of surface plasmon (SP) on a metal structure with a light emitter can enhance its emission efficiency. Such a coupling behavior for emission enhancement has also been observed in an InGaN/GaN quantum well (QW) structure. ${ }^{1-9}$ In such a coupling process, energy in the excited carriers in the $\mathrm{QW}$ is first transferred into the SP modes on a metal structure nearby. The SP modes can radiate effectively if the momentum mismatch between SPs and photons can be compensated. Such a compensation scheme can be obtained from a metal grating structure or a rough metal surface. For a certain SP mode, a metal grating of an appropriate period for momentum matching can significantly enhance the SP radiation efficiency. Such momentum matching can be satisfied only for the SP component propagating in the direction perpendicular to the grating grooves. Therefore, the SP-QW coupling process in a light-emitting diode with a onedimensional (1D) metal grating structure can lead to polarized output, ${ }^{10}$ which is useful for backlighting applications of liquid-crystal display.

In this letter, we report the observation of polarizationdependent SP-QW coupling behavior in an InGaN/GaN QW structure with a $1 \mathrm{D}$ Ag grating. In the recorded PL intensity distribution in the energy-in-plane-wavevector $\left(E-k_{x}\right)$ space, which includes both the diffraction/interference fringes of the grating and the SP features, the SP feature is identified from the polarization-dependent measurement, the calibrated SP group velocity, and the shift of SP dispersion curve in changing grating period. The used InGaN/GaN QW epitaxial sample includes two QWs for individually emitting blue and green lights. The growth of the two QWs extends the

${ }^{a)}$ Tel.: 886-2-23657624. FAX: 886-2-23652637. Electronic mail: ccy@cc.ee.ntu.edu.tw. emission coverage for easier identification of SP dispersion curve. The two QWs and the $2 \mu \mathrm{m}$ undoped GaN lowercladding layer are grown on $c$-plane sapphire substrate with metal-organic chemical vapor deposition. The widths of the barrier between the blue QW (deeper) and green QW and the cap layer above the green $\mathrm{QW}$, both undoped $\mathrm{GaN}$, are 10 and $15 \mathrm{~nm}$, respectively. Therefore, the $3 \mathrm{~nm}$ green and blue QWs are 15 and $28 \mathrm{~nm}$ below the $\mathrm{Ag} / \mathrm{GaN}$ interface, respectively. The 1D Ag grating patterns were defined by electronbeam lithography. A Ag layer of $50 \mathrm{~nm}$ in thickness was deposited on the epitaxial sample by electron-gun evaporation. A lift-off procedure was performed for forming the grating grooves. The periods of $\mathrm{Ag}$ gratings are varied from 300 to $450 \mathrm{~nm}$ with a step size of $50 \mathrm{~nm}$ (four samples in total). The duty cycle of the gratings was fixed at $50 \%$. In other words, on each sample, one-half of semiconductor surface is exposed to air. Figure 1 shows a plan-view scanning electron microscopy (SEM) image of a Ag grating with $400 \mathrm{~nm}$ in period. The coordinate system is also defined in this figure.

In photoluminescence (PL) measurement, PL is excited by a $406 \mathrm{~nm}$ InGaN laser diode from the bottom side (sapphire side). The angle-dependent PL intensity is recorded also from the bottom side. The excitation laser is focused with a convex lens of $100 \mathrm{~mm}$ in focal length and incident onto the sample in the $y-z$ plane through a fused-silica hemispherical lens of $4 \mathrm{~mm}$ in diameter. The hemispherical lens provides the function of solid immersion. ${ }^{11}$ It is attached to the bottom side of the sample for increasing excitation intensity and PL photon collection efficiency in the PL measurement. Index-matching oil is introduced to the interface between the hemispherical lens and the sapphire substrate to reduce the totally internal reflection from the air gap. The use of the hemispherical lens also increases the measurement 


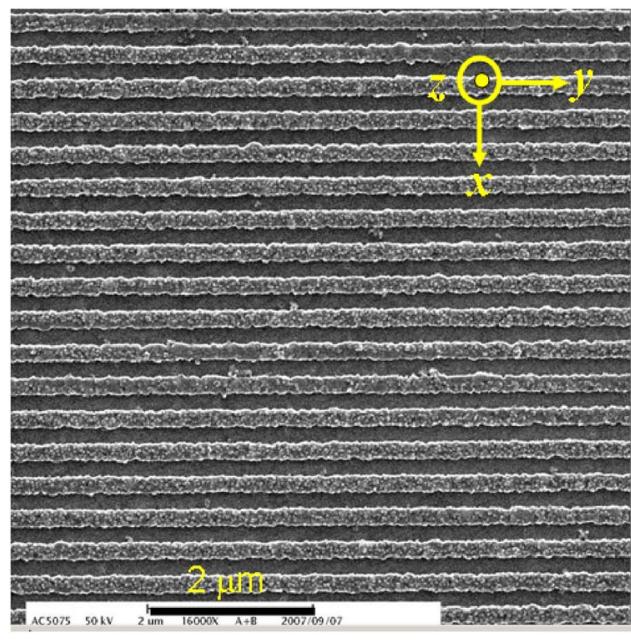

FIG. 1. (Color online) SEM image of a Ag grating with $400 \mathrm{~nm}$ in period.

range of $k_{x}$ by a factor of $n_{\text {eff }}$, where $n_{\text {eff }}$ is the effective refractive index of the combination of the hemispherical lens and the sapphire substrate. Because the thickness of the sapphire substrate $(<150 \mu \mathrm{m}$ after thinning $)$ is much smaller than the diameter of the hemispherical lens, $n_{\text {eff }}$ can be reasonably approximated by the refractive index of the hemispherical lens. PL photons are collected by a detection unit, consisting of a variable iris, a focal lens, and an optical fiber, from the bottom side of the sample through the hemispherical lens. The detection unit is mounted on a computercontrolled motorized rotary stage. It is rotated around the grating sample in the $x-z$ plane.

Figure 2 shows the $x-z$ polarized ( $p$-polarized) PL intensity distributions in the $E-k_{x}$ space when the Ag grating periods are $300 \mathrm{~nm}$ (a), $350 \mathrm{~nm}$ (b), $400 \mathrm{~nm} \mathrm{(c),} \mathrm{and} 450 \mathrm{~nm}$ (d). Here, $k_{x}$ is related to the measurement angle $\theta$ through the relation

$$
k_{x}(\lambda, \theta)=2 \pi \sin \theta \cdot n_{\mathrm{eff}}(\lambda) / \lambda .
$$

Here, $\theta$ is the rotation angle of the detection unit from the $z$ axis. In these figures, one can see two bright bands from the blue- and green-emitting QWs. The (red) dashed lines labeled "SP" show the dispersion relation of the SP mode, which is to be discussed later. Other dashed lines labeled A-C represent the cutoff point of the -1 diffraction order of
TABLE I. Grating diffraction effects in various samples of different grating periods.

\begin{tabular}{cl}
\hline Labeled features in Figs. 2 and 3 & Descriptions \\
\hline Fringe 1 & $\begin{array}{l}\text { Interference between the }-1 \text { st and }+1 \text { st } \\
\text { diffraction components } \\
\text { Interference between the 0th and }-1 \text { st } \\
\text { diffraction components }\end{array}$ \\
Fringes 2, 3, 6, 8, and 11 & $\begin{array}{l}\text { Interference between the 0th and +2nd } \\
\text { diffraction components }\end{array}$ \\
Fringes 4, 9, and 10 & $\begin{array}{l}\text { Interference between the }-1 \text { th and }+2 \text { nd } \\
\text { diffraction components } \\
\text { Cutoff of the }-1 \text { st diffraction }\end{array}$ \\
Fringes 5 and 7 &
\end{tabular}

the grating (see Table I). In the PL intensity distributions, one can clearly see various fine fringe patterns at different $E-k_{x}$ coordinates, as labeled with various numbers. The common large-period fringe in all figures of Fig. 2 originates from the Fabry-Pérot effect of the sample, i.e., the oscillation between the metal/semiconductor and semiconductor/sapphire interfaces. The diffraction effects of the metal grating are summarized in Table I, including the origins of those fine fringes (labels 1-9) and the lines for the cutoff of a diffraction order (labels A-C). Those fine fringes come from the interference of various grating diffraction orders. However, they are not of our major concern in this study. In each part of Fig. 2, the vertical arrow around $1.7 \mu \mathrm{m}^{-1}$ in $k_{x} / 2 \pi$ indicates an almost vertical dark band, which corresponds to the Brewster's angle effect at the GaN/sapphire interface in the $p$-polarized case. Other dark bands are caused by measurement instability.

In Fig. 2, the dashed lines labeled SP connect the bright spots in the blue and green bands of the PL intensity distributions. They correspond to a section of the dispersion curve of a SP mode propagating in the air $/ \mathrm{Ag} / \mathrm{GaN}$ grating structure. ${ }^{12,13}$ Since the expected SP resonance energy of the grating structure is significantly higher than the energy range in Fig. 2, it is reasonable to fit the dispersion relation with a linear curve. The SP resonance energy is estimated to be larger than $3.4 \mathrm{eV}$ based on a coupled-wave-method calculation. The SP group velocity can be estimated from the fitted SP dispersion curve to give about $1 \mathrm{eV} \mu \mathrm{m}$ or 2.42 $\times 10^{8} \mathrm{~m} / \mathrm{s}$ within the emission energy range of the dual QWs. This group velocity is quite consistent with that of
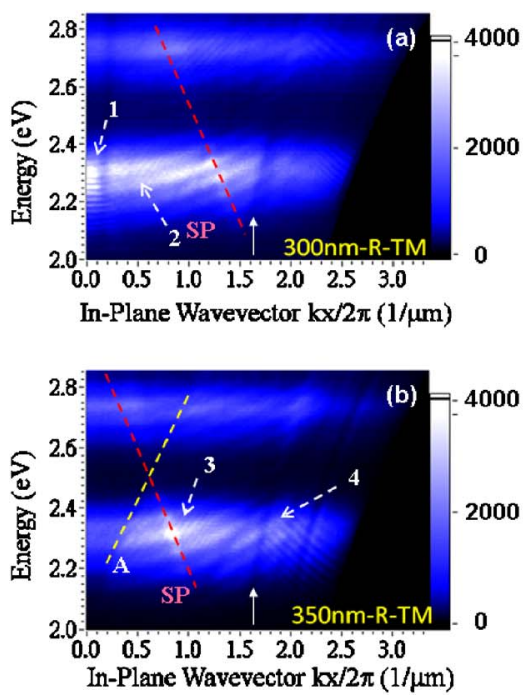
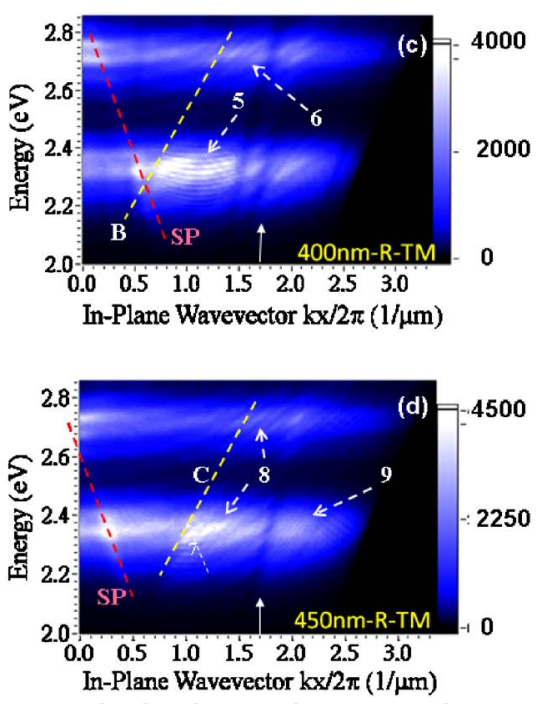

FIG. 2. (Color online) Distributions of the $p$-polarized PL intensity in the $E-k_{x}$ space when the metal grating periods are $300 \mathrm{~nm} \mathrm{(a),} 350 \mathrm{~nm} \mathrm{(b),}$ $400 \mathrm{~nm}(\mathrm{c})$, and $450 \mathrm{~nm}(\mathrm{~d})$. 

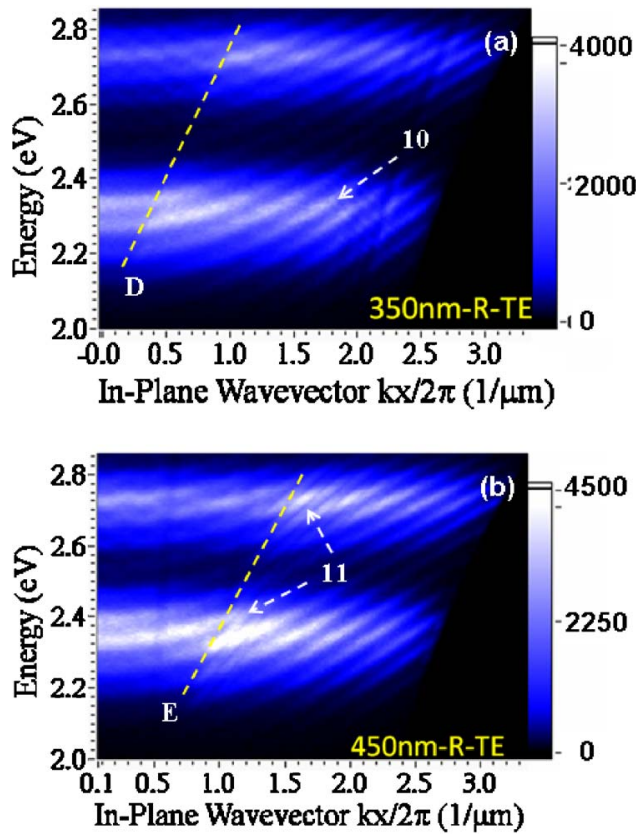

FIG. 3. (Color online) Distributions of the $s$-polarized PL intensity in the $E-k_{x}$ space when the metal grating periods are $350 \mathrm{~nm}$ (a) and $450 \mathrm{~nm}$ (b).

around $2.61 \times 10^{8} \mathrm{~m} / \mathrm{s}$ from a numerical study. The large group velocity represents a SP feature in the air/ $\mathrm{Ag} / \mathrm{GaN}$ grating structure. The observed dispersion curve belongs to the negative $k_{x}$ range and is right shifted to the positive side through the metal grating coupling. Therefore, the relative positions in the $k_{x}$ axis of the four SP lines in Figs. 2(a) -2 (d) are related to their individual grating periods. The differences between the interception points in the $k_{x}$ (divided by $2 \pi$ ) axis of the four SP lines in Fig. 2 are $0.46,0.34$, and $0.25 \mu \mathrm{m}^{-1}$. They are close to the differences of the inverse grating period between two successive periods as $1 / 0.3-1 / 0.35=0.476 \mu \mathrm{m}^{-1}, \quad 1 / 0.35-1 / 0.4=0.357 \mu \mathrm{m}^{-1}$, and $1 / 0.4-1 / 0.45=0.278 \mu \mathrm{m}^{-1}$, respectively. The general consistency of those numbers strongly supports the observation of a propagating SP mode in the air/ $\mathrm{Ag} / \mathrm{GaN}$ grating structure.

The other result supporting the observation of the SP mode is given in Fig. 3, in which the $y$-polarized $(s$-polarized) bottom-side PL intensity distributions in the $E-k_{x}$ space of the samples with the grating periods of $350 \mathrm{~nm}$ (a) and $450 \mathrm{~nm}$ (b) are shown. In these figures, the largeperiod Fabry-Pérot fringes, a few fine fringes caused by the interference of grating diffraction orders (labels 10 and 11), and two dashed lines (labeled with D and E) for showing the cutoff of grating diffraction can be seen. Their grating diffraction information is also shown in Table I. The key point in Fig. 3 and the similar data (in the cases of 300 and $400 \mathrm{~nm}$ grating periods) is the absence of the corresponding SP dispersion curve. We also performed the polarization-dependent PL measurement by rotating the grating sample by $90^{\circ}$ with respect to the $z$ axis. In this situation, no SP feature was observed for both $p$ - and $s$-polarized measurements. This result is attributed to the lack of momentum compensation from the 1D grating in this orientation.

In summary, we have reported the observation of a polarization-dependent SP feature on a 1D Ag grating structure through the SP coupling with an InGaN/GaN dual-QW structure below the metal grating. The angle- and polarization-dependent PL measurements demonstrated the polarized SP characteristics in such a $1 \mathrm{D}$ air $/ \mathrm{Ag} / \mathrm{GaN}$ grating structure. The SP group velocity was estimated to be around $2.42 \times 10^{8} \mathrm{~m} / \mathrm{s}$.

This research was supported by National Science Council, The Republic of China, under Grant No. NSC 96-2120M-002-008 and NSC 96-2628-E-002-044-MY3, and by US Air Force Scientific Research Office under Contract No. AOARD-07-4010.

${ }^{1}$ K. Okamoto, I. Niki, A. Shvartser, Y. Narukawa, T. Mukai, and A. Scherer, Nat. Mater. 3, 601 (2004).

${ }^{2}$ K. Okamoto, I. Niki, A. Scherer, Y. Narukawa, T. Mukai, and Y. Kawakami, Appl. Phys. Lett. 87, 071102 (2005).

${ }^{3}$ G. Sun, J. B. Khurgin, and R. A. Soref, Appl. Phys. Lett. 90, 111107 (2007).

${ }^{4}$ J. B. Khurgin, G. Sun, and R. A. Soref, J. Opt. Soc. Am. B 24, 1968 (2007).

${ }^{5}$ C. Y. Chen, D. M. Yen, Y. C. Lu, and C. C. Yang, Appl. Phys. Lett. 89, 203113 (2006).

${ }^{6}$ C. Y. Chen, Y. C. Lu, D. M. Yeh, and C. C. Yang, Appl. Phys. Lett. 90, 183114 (2007).

${ }^{7}$ Y. C. Lu, C. Y. Chen, D. M. Yeh, C. F. Huang, T. Y. Tang, J. J. Huang, and C. C. Yang, Appl. Phys. Lett. 90, 193103 (2007).

${ }^{8}$ D. M. Yeh, C. Y. Chen, Y. C. Lu, C. F. Huang, and C. C. Yang, Nanotechnology 18, 265402 (2007).

${ }^{9}$ D. M. Yeh, C. F. Huang, Y. C. Lu, C. Y. Chen, T. Y. Tang, J. J. Huang, K. C. Shen, Y. J. Yang, and C. C. Yang, Appl. Phys. Lett. 91, 063121 (2007).

${ }^{10}$ P. T. Worthing and W. L. Barnes, J. Mod. Opt. 49, 1453 (2002).

${ }^{11}$ S. Moehl, H. Zhao, B. D. Don, S. Wachter, and H. Kalt, J. Appl. Phys. 93, 6265 (2003).

${ }^{12}$ C. Bonnand, J. Bellessa, C. Symonds, and J. C. Plenet, Appl. Phys. Lett. 89, 231119 (2006).

${ }^{13}$ U. Schroter and D. Heitman, Phys. Rev. B 60, 4992 (1999). 